\title{
SHORTFALL RISK APPROXIMATIONS FOR AMERICAN OPTIONS IN THE MULTIDIMENSIONAL BLACK-SCHOLES MODEL
}

\author{
YAN DOLINSKY, ${ }^{*}$ ETH Zürich
}

\begin{abstract}
We show that shortfall risks of American options in a sequence of multinomial approximations of the multidimensional Black-Scholes (BS) market converge to the corresponding quantities for similar American options in the multidimensional BS market with path-dependent payoffs. In comparison to previous papers we consider the multiassets case for which we use the weak convergence approach.
\end{abstract}

Keywords: American option; shortfall risk; weak convergence

2010 Mathematics Subject Classification: Primary 91B28

Secondary 60F05

\section{Introduction}

In this paper we deal with multinomial approximations of the shortfall risk for American options in the multidimensional Black-Scholes (BS) (complete) model. It is well known that in a complete market an American contingent claim can be hedged perfectly with an initial capital which is equal to the optimal stopping value of the discounted payoff under the unique martingale measure. In real market conditions an investor (seller) may not be willing for various reasons to tie in a hedging portfolio the full initial capital required for a perfect hedge. In this case the seller is ready to accept a risk that his portfolio value at an exercise time may be less than his obligation to pay and he will need additional funds to fulfill the contract. Thus, a portfolio shortfall comes into the picture.

We deal with a certain type of risk called the shortfall risk, which is defined as the maximal expectation (with respect to the buyer exercise times) of the discounted shortfall (see [13]). An investor whose initial capital is less than the option price still wants to compute the minimal possible shortfall risk and to find a portfolio strategy which minimizes or 'almost' minimizes the shortfall risk. In this paper we allow only admissible self financing portfolios, i.e. portfolios with nonnegative wealth process. This corresponds to the situation when the portfolio is handled without borrowing of the capital.

For discrete-time markets such as the multinomial models, the above problems can be solved by a dynamical programming algorithm. For continuous-time models such as the BS model, these problems are much more complicated.

We prove that, for American options, the shortfall risk in the multidimensional BS model can be approximated by a sequence of shortfall risks in an appropriate multinomial model. This type of result has a practical value since the shortfall risks in the multinomial models can be calculated via a dynamical programming algorithm. Our main tools are the extended weak

Received 5 February 2010; revision received 20 August 2010.

* Postal address: Department of Mathematics, ETH Zürich, 8092 Zürich, Switzerland.

Email address: yan.dolinsky@math.ethz.ch 
convergence theory that was developed in [1] and the tightness theorems that were obtained in [12]. Since we use the weak convergence approach, we could not provide error estimates of the above approximations. Thus, two problems remain open. The first problem is to obtain error estimates of the above approximations. The second problem is to find explicit formulae for optimal or 'almost' optimal hedges in the BS model. It seems that both of the above problems require new tools.

To date, shortfall risk approximations have been studied only in the one-dimensional BS model (see [5] and [6]). For this case, it was proved that the shortfall risk in a BS market is a limit of the shortfall risks in an appropriate sequence of Cox-Ross-Rubinstein (CRR) markets. Furthermore, the authors obtained error estimates and a dynamical programming algorithm for 'almost' optimal hedges. The main tool that was used in the above papers was the Skorokhod embedding tool of independent and identically distributed random variables into one-dimensional Brownian motion. This tool cannot be applied to multidimensional Brownian motion.

The main results of this paper are formulated in the next section. In Section 3 we derive auxiliary lemmas that will be essential in the proof of the main result. In Section 4 we complete the proof of the main result of the paper. In Section 5 we analyze the multinomial models and provide a dynamical programming algorithm for the shortfall risk and the corresponding optimal portfolios.

\section{Preliminaries and main results}

First we introduce the multidimensional BS market. Consider a complete probability space $\left(\Omega_{W}, \mathrm{P}^{W}\right)$ together with a standard $d$-dimensional continuous in time Brownian motion $\left\{W(t)=\left(W_{1}(t), \ldots, W_{d}(t)\right)\right\}_{t=0}^{\infty}$ and filtration $\mathcal{F}_{t}^{W}=\sigma\{W(s) \mid s \leq t\}$. We assume that the $\sigma$-algebras contain the null sets. A BS financial market consists of a savings account $B(t)$ with an interest rate $r$, assuming without loss of generality that $r=0$, i.e.

$$
B(t)=B(0)>0,
$$

and of $d$ risky stocks $S^{W}=\left(S_{1}^{W}, \ldots, S_{d}^{W}\right)$ given by

$$
S_{i}^{W}(t)=S_{i}(0) \exp \left(\sum_{j=1}^{d} \sigma_{i j} W_{j}(t)+\left(b_{i}-\frac{1}{2} \sum_{j=1}^{d} \sigma_{i j}^{2}\right) t\right), \quad S_{i}(0)>0,
$$

where $b \in \mathbb{R}^{d}$ is a constant vector and $\sigma \in M_{d}(\mathbb{R})$ is a constant nonsingular matrix.

Let $T<\infty$ be the maturity date of our American option, and let $\mathcal{T}_{[0, T]}^{W}$ be the set of all stopping times with respect to $\mathcal{F}^{W}$ which take values in $[0, T]$. Denote by $\left(\mathbb{D}\left([0, T] ; \mathbb{R}^{d}\right), \S\right)$ the space of all càdlàg functions, i.e. right-continuous functions with left-hand limits, equipped with the Skorokhod topology (see [2, Chapter 3]). Let $F:[0, T] \times\left(\mathbb{D}\left([0, T] ; \mathbb{R}^{d}\right), \S\right) \rightarrow \mathbb{R}_{+}$ be measurable functions such that there exists a constant $C>0$ which satisfies

$$
\sup _{0 \leq t \leq T} F(t, x) \leq C \sup _{0 \leq t \leq T}|x(t)| \quad \text { for all } x \in \mathbb{D}\left([0, T] ; \mathbb{R}^{d}\right) .
$$

Furthermore, we assume that, for any $t \in[0, T]$ and $x, y \in \mathbb{D}\left([0, T] ; \mathbb{R}^{d}\right)$,

(i) $F(\cdot, x)$ is a right-continuous function with left-hand limits;

(ii) $F(t, x)=F(t, y)$ if $x(s)=y(s)$ for any $s \leq t$;

(iii) if $x$ is continuous at $t$ then $F$ is continuous at $(x, t)$ (with respect to the product topology). 
Next, consider an American option with a payoff process given by

$$
Y^{W}(t)=F\left(t, S^{W}\right), \quad 0 \leq t \leq T
$$

From the assumptions above, it follows that $\left\{Y^{W}(t)\right\}_{t=0}^{T}$ is a càdlàg adapted stochastic process and that $\mathrm{E}^{W}\left[\sup _{0 \leq t \leq T} Y^{W}(t)\right]<\infty$. Denote by $\tilde{\mathrm{P}}^{W}$ the unique martingale measure for the above model. Using standard arguments, it follows that the restriction of the probability measure $\tilde{\mathrm{P}}^{W}$ to the $\sigma$-algebra $\mathcal{F}_{t}^{W}$ satisfies

$$
M(t)=\frac{\mathrm{d} \tilde{\mathrm{P}}^{W}}{\mathrm{dP}^{W}} \mid \mathcal{F}_{t}^{W}=\exp \left(-\frac{1}{2}\|\theta\|^{2} t-\langle\theta, W(t)\rangle\right),
$$

where $\theta=b\left(\sigma^{\top}\right)^{-1}$. Here $\|\cdot\|$ and $\langle\cdot, \cdot\rangle$ denote the standard norm and the scalar product of $\mathbb{R}^{d}$, respectively.

A self-financing strategy $\pi$ with a horizon $T$ and an initial capital $x$ (see [15, Chapter 7]) is a $d$-dimensional progressively measurable process $\pi=\{\gamma(t)\}_{t=0}^{T}$ which satisfies

$$
\int_{0}^{T}\left\langle\gamma(t), S^{W}(t)\right\rangle^{2} \mathrm{~d} t<\infty \quad \text { almost surely (a.s.). }
$$

For a strategy $\pi$, the portfolio value process $\left\{V^{\pi}(t)\right\}_{t=0}^{T}$ is given by

$$
V^{\pi}(t)=x+\int_{0}^{t}\left\langle\gamma(u), \mathrm{d} S^{W}(u)\right\rangle .
$$

Recall (see [11]) that stochastic integrals with respect to the Brownian motion have a continuous modification and so, for any self-financing strategy $\pi$, the corresponding portfolio value process is a continuous process.

A self-financing strategy $\pi$ is called admissible if $V^{\pi}(t) \geq 0$ for all $t \in[0, T]$; the set of such strategies with an initial capital no bigger than $x$ will be denoted by $\mathcal{A}^{W}(x)$. We set $A^{W}=\bigcup_{x>0} A^{W}(x)$. For an admissible self-financing strategy $\pi$, the shortfall risk is given by (see [13])

$$
R(\pi)=\sup _{\tau \in \mathcal{T}_{[0, T]}^{W}} \mathrm{E}^{W}\left[\left(Y^{W}(\tau)-V^{\pi}(\tau)\right)^{+}\right],
$$

which is the maximal possible expectation with respect to the probability measure $\mathrm{P}^{W}$ of the (discounted) shortfall. The shortfall risk for an initial capital $x$ is given by

$$
R(x)=\inf _{\pi \in \mathcal{A}^{W}(x)} R(\pi) .
$$

Next, we introduce the sequence of multinomial markets that we use to approximate the shortfall risk in the BS model. The same markets were used in [8] in order to approximate European option prices in the $d$-dimensional BS model. Let $A \in M_{d+1}(\mathbb{R})$ be an orthogonal matrix such that its last column equals $(1 / \sqrt{d+1}, \ldots, 1 / \sqrt{d+1})^{\top}$. Let $\Omega_{\xi}=\{1,2, \ldots, d+1\}^{\infty}$ be the space of finite sequences $\omega=\left(\omega_{1}, \omega_{2}, \ldots\right) ; \omega_{i} \in\{1,2, \ldots, d+1\}$ with the product probability $\mathrm{P}^{\xi}=\{1 /(d+1), \ldots, 1 /(d+1)\}^{\infty}$. Define a sequence of independent and identically distributed random vectors $\xi^{(1)}, \xi^{(2)}, \ldots$ by

$$
\xi^{(i)}(\omega)=\sqrt{d+1}\left(A_{\omega_{i} 1}, A_{\omega_{i} 2}, \ldots, A_{\omega_{i} d}\right), \quad i \in \mathbb{N} .
$$


Let $\mathcal{F}_{m}^{\xi}=\sigma\left\{\xi^{(k)} \mid k \leq m\right\}, m \geq 0\left(\mathcal{F}_{0}^{\xi}=\left\{\varnothing, \Omega_{\xi}\right\}\right)$. Denote by $\mathcal{T}_{m}^{\xi}$ the set of all stopping times with respect to the filtration $\left\{\mathcal{F}_{k}^{\xi}\right\}_{k=0}^{\infty}$ with values from 0 to $m$.

For any $n$, consider the $n$-step multinomial market which consists of a savings account $B^{(n)}(t)$ given by

$$
B^{(n)}(t)=B(0)>0
$$

and of $d$ risky stocks $S^{\xi, n}=\left(S_{1}^{\xi, n}, \ldots, S_{d}^{\xi, n}\right)$ given by the formulae $S_{i}^{\xi, n}(t)=S_{i}(0)$ for $t \in$ $[0, T / n)$ and

$$
S_{i}^{\xi, n}(t)=S_{i}(0) \prod_{m=1}^{k}\left(1+\frac{b_{i} T}{n}+\sqrt{\frac{T}{n}} \sum_{j=1}^{d} \sigma_{i j} \xi_{j}^{(m)}\right), \quad \frac{k T}{n} \leq t<\frac{(k+1) T}{n}, k=1, \ldots, n .
$$

We assume that $n$ is sufficiently large such that the terms in the above product are positive a.s. The market is active at the times $0, T / n, 2 T / n, \ldots, T$. It is well known that this market is complete, and we denote by $\tilde{\mathrm{P}}_{n}^{\xi}$ the unique martingale measure. Define the stochastic process $\left\{M^{(n)}(t)\right\}_{t=0}^{T}$ by

$$
M^{(n)}(t)=\frac{\mathrm{d} \tilde{\mathrm{P}}_{n}^{\xi}}{\mathrm{dP} \xi} \mid \mathcal{F}_{k}^{\xi}, \quad \frac{k T}{n} \leq t<\frac{(k+1) T}{n}, k=0,1, \ldots, n .
$$

Clearly $\left\{M^{(n)}(k T / n)\right\}_{k=0}^{n}$ is a martingale with respect to the probability measure $\mathrm{P}^{\xi}$ and the filtration $\left\{\mathcal{F}_{k}^{\xi}\right\}_{k=0}^{n}$. Explicit formulae for $M^{(n)}(t)$ were obtained in [8]. Consider an American option with the adapted payoff process

$$
Y^{\xi, n}(k)=F\left(\frac{k T}{n}, S^{\xi, n}\right), \quad 0 \leq k \leq n .
$$

A self-financing strategy $\pi$ with an initial capital $x$ and a horizon $n$ (see [15, Chapter 5]) is a sequence $\pi=(\gamma(1), \ldots, \gamma(n))$, where the $\gamma(k)$ are $\mathcal{F}_{k-1}^{\xi}$-measurable random vectors. The portfolio value $V^{\pi}(k), k=0,1, \ldots, n$, is given by

$$
V^{\pi}(k)=x+\sum_{i=0}^{k-1}\left\langle\gamma(i+1),\left(S^{\xi, n}\left(\frac{(i+1) T}{n}\right)-S^{\xi, n}\left(\frac{i T}{n}\right)\right)\right\rangle .
$$

We call a self-financing strategy $\pi$ admissible if $V^{\pi}(k) \geq 0$ for any $k \leq n$. Denote by $\mathcal{A}^{\xi, n}(x)$ the set of all admissible self-financing strategies with an initial capital no bigger than $x$, and let $\mathscr{A}^{\xi, n}=\bigcup_{x>0} \mathcal{A}^{\xi, n}(x)$. The definitions for the shortfall risks in the multinomial markets are similar to the definitions in the BS model. Thus, for the $n$-step multinomial market, the shortfall risks are given by

$$
R_{n}(\pi)=\max _{\tau \in \mathcal{T}_{n}^{\xi}} \mathrm{E}^{\xi}\left[\left(Y^{\xi, n}(\tau)-V^{\pi}(\tau)\right)^{+}\right] \quad \text { and } \quad R_{n}(x)=\inf _{\pi \in \mathcal{A}^{\xi, n}(x)} R_{n}(\pi),
$$

where $\mathrm{E}^{\xi}$ is the expectation with respect to $\mathrm{P}^{\xi}$.

The following theorem is the main result of the paper and it says that the shortfall risk $R(x)$ for an initial capital $x$ of an American option in the multidimensional BS market can be approximated by a sequence of shortfall risks with an initial capital $x$ of American options in the multinomial markets defined above. This result has a practical value since, for any $n$, the shortfall risk $R_{n}(x)$ can be calculated via a dynamical programming algorithm, which is given in Section 5. 
Theorem 2.1. For any $x>0$,

$$
\lim _{n \rightarrow \infty} R_{n}(x)=R(x) .
$$

The proof (which is given in Section 4) consists of two parts. In the first part we prove that the inequality $R(x) \leq \liminf _{n \rightarrow \infty} R_{n}(x)$ holds and in the second part we prove that $R(x) \geq \lim \sup _{n \rightarrow \infty} R_{n}(x)$ holds. In the first part we take a sequence of 'almost' optimal portfolios $\left\{\pi_{n}\right\}_{n=1}^{\infty}$ for the multinomial markets and consider their limit in some sense that will be explained explicitly in Section 3. From the limit process we construct a portfolio $\pi$ in the BS model such that $R(\pi) \leq \liminf _{n \rightarrow \infty} R_{n}\left(\pi_{n}\right)=\liminf _{n \rightarrow \infty} R_{n}(x)$. The second part is proved by a reversed operation. Namely, we take an 'almost' optimal portfolio $\pi$ in the BS model which has some smoothness properties. The existence of such a portfolio will be proved by applying density arguments. From this portfolio we construct a sequence of portfolios $\left\{\pi_{n}^{\prime}\right\}_{n=1}^{\infty}$ in the multinomial models which satisfies $\lim _{\sup _{n \rightarrow \infty}} R_{n}\left(\pi_{n}^{\prime}\right) \leq R(\pi)$.

\section{Auxiliary lemmas}

Let $I \subset[0, T]$ be a dense set in $[0, T]$, and let $\mathcal{T}_{I} \subset \mathcal{T}_{[0, T]}^{W}$ be the set of all stopping times with a finite number of values which belongs to $I$.

Lemma 3.1. For any $\pi \in \mathcal{A}^{W}$,

$$
R(\pi)=\sup _{\tau \in \mathcal{T}_{I}} \mathrm{E}^{W}\left[\left(Y^{W}(\tau)-V^{\pi}(\tau)\right)^{+}\right]
$$

Proof. Choose $\varepsilon>0$. There exists a $\tau \in \mathcal{T}_{[0, T]}^{W}$ such that

$$
R(\pi)<\mathrm{E}^{W}\left[\left(Y^{W}(\tau)-V^{\pi}(\tau)\right)^{+}\right]+\varepsilon
$$

For any $n$, there exists a finite set $I_{n} \subset I$ for which $\bigcup_{z \in I_{n}}(z-1 / n, z+1 / n) \supseteq[0, T]$. Let $a_{n}$ be the maximal element of $I_{n}$. Define $\tau_{n}=\min \left\{t \in I_{n} \mid t \geq \tau\right\} \mathbf{1}_{\left\{\tau_{n} \leq a_{n}\right\}}+a_{n} \mathbf{1}_{\left\{\tau_{n}>a_{n}\right\}}$, where $\mathbf{1}_{D}=1$ if an event $D$ occurs and $\mathbf{1}_{D}=0$ otherwise. Clearly, $\tau_{n} \leq a_{n}$ a.s. and, for $t \in I_{n} \backslash\left\{a_{n}\right\}$, we have $\left\{\tau_{n} \leq t\right\}=\{\tau \leq t\} \in \mathcal{F}_{t}^{W}$. Thus, $\tau_{n} \in \mathcal{T}_{I}$. Furthermore, $\left|\tau_{n}-\tau\right| \leq 2 / n$ and so $\tau_{n} \rightarrow \tau$ a.s. From (3.1) and the assumptions on $F$, we obtain

$$
\begin{aligned}
R(\pi) & <\varepsilon+\mathrm{E}^{W}\left[\lim _{n \rightarrow \infty}\left(Y^{W}\left(\tau_{n}\right)-V^{\pi}\left(\tau_{n}\right)\right)^{+}\right] \\
& =\varepsilon+\lim _{n \rightarrow \infty} \mathrm{E}^{W}\left[\left(Y^{W}\left(\tau_{n}\right)-V^{\pi}\left(\tau_{n}\right)\right)^{+}\right] \\
& \leq \varepsilon+\sup _{\tau \in \mathcal{T}_{I}} \mathrm{E}^{W}\left[\left(Y^{W}(\tau)-V^{\pi}(\tau)\right)^{+}\right],
\end{aligned}
$$

and the result follows by letting $\varepsilon \downarrow 0$.

The next lemma provides a general result for the shortfall risk measure.

Lemma 3.2. Let $x>0$. For any $\varepsilon>0$, there exists $0 \leq \psi \in C\left(\left(\mathbb{D}\left([0, T] ; \mathbb{R}^{d}\right)\right.\right.$, s) ) such that the martingale given by $Q(t)=\mathrm{E}^{W}\left[\psi\left(S^{W}\right) \mid \mathcal{F}_{t}^{W}\right], t \leq T$, satisfies

$$
Q(0)<x \text { and } R(x)>\sup _{\tau \in \mathcal{T}_{[0, T]}^{W}} \mathrm{E}^{W}\left[\left(Y^{W}(\tau)-\frac{Q(\tau)}{M(\tau)}\right)^{+}\right]-\varepsilon
$$


Proof. Let $\varepsilon>0$. Set $K=\mathrm{E}^{W}\left[\sup _{0 \leq t \leq T} 1 / M(t)\right]<\infty$ and $\delta=\varepsilon /(2(K+1))$. There exists a $\pi \in \mathcal{A}(x)$ such that $R(\pi)<R(x)+\delta$. The process $\Phi(t):=V^{\pi}(t) M(t), t \leq T$, is a supermartingale with respect to $\mathrm{P}^{W}$. Introduce the regular martingale

$$
\Gamma(t)=\mathrm{E}^{W}\left[\sup _{0 \leq u \leq T} Y^{W}(u) M(u) \mid \mathcal{F}_{t}^{W}\right], \quad t \leq T .
$$

The process $\Psi(t):=\Phi(t) \wedge \Gamma(t)$ is a supermartingale of class $\mathscr{D}$. By Doob's decomposition theorem, there exists a continuous martingale $\{U(t)\}_{t=0}^{T}$ such that $U(0)=\Psi(0) \leq \Phi(0)=x$ and $U(t) \geq \Psi(t)$ a.s. for all $t \leq T$. Clearly, $\Gamma(t) \geq Y^{W}(t) M(t)$, and so

$$
\left(Y^{W}(t)-\frac{\Psi(t)}{M(t)}\right)^{+}=\left(Y^{W}(t)-V^{\pi}(t)\right)^{+} \text {a.s. }
$$

for all $t \leq T$. Thus,

$$
\begin{aligned}
\sup _{\tau \in \mathcal{T}_{[0, T]}^{W}} \mathrm{E}^{W}\left[\left(Y^{W}(\tau)-\frac{U(\tau)}{M(\tau)}\right)^{+}\right] & \leq \sup _{\tau \in \mathcal{T}_{[0, T]}^{W}} \mathrm{E}^{W}\left[\left(Y^{W}(\tau)-\frac{\Psi(\tau)}{M(\tau)}\right)^{+}\right] \\
& =\sup _{\tau \in \mathcal{T}_{[0, T]}^{W}} \mathrm{E}^{W}\left[\left(Y^{W}(\tau)-V^{\pi}(\tau)\right)^{+}\right] \\
& <R(x)+\delta .
\end{aligned}
$$

Next, choose a sequence $0 \leq \psi_{n} \in C\left(\left(\mathbb{D}\left([0, T] ; \mathbb{R}^{d}\right), \delta\right)\right), n \geq 1$, such that

$$
\lim _{n \rightarrow \infty} \mathrm{E}^{W}\left|\psi_{n}\left(S^{W}\right)-U(T)\right|=0 \quad \text { and } \quad \mathrm{E}^{W} \psi_{n}\left(S^{W}\right)<\mathrm{E}^{W} U(T) \leq x, \quad n \in \mathbb{N} .
$$

Set $Q^{(n)}(t)=\mathrm{E}^{W}\left[\psi_{n}\left(S^{W}\right) \mid \mathcal{F}_{t}^{W}\right], t \leq T$, and introduce the set $C_{n}=\left\{\sup _{0 \leq t \leq T} \mid U(t)\right.$ $-Q^{(n)}(t) \mid>\delta$. From (3.3) we obtain, for any $n$,

$$
\begin{aligned}
\sup _{\tau \in \mathcal{T}_{[0, T]}^{W}} & \mathrm{E}^{W}\left[\left(Y^{W}(\tau)-\frac{Q^{(n)}(\tau)}{M(\tau)}\right)^{+}\right] \\
\leq & \sup _{\tau \in \mathcal{T}_{[0, T]}^{W}} \mathrm{E}^{W}\left[\left(Y^{W}(\tau)-\frac{U(\tau)}{M(\tau)}\right)^{+}\right]+\delta \mathrm{E}^{W}\left[\sup _{0 \leq t \leq T} \frac{1}{M(t)}\right] \\
& +\mathrm{E}^{W}\left[\mathbf{1}_{C_{n}} \sup _{0 \leq t \leq T} Y^{W}(t)\right] \\
< & R(x)+\frac{\varepsilon}{2}+\mathrm{E}^{W}\left[\mathbf{1}_{C_{n}} \sup _{0 \leq t \leq T} Y^{W}(t)\right] .
\end{aligned}
$$

By using Doob's inequality for the continuous submartingale $\left\{\left|U(t)-Q^{(n)}(t)\right|\right\}_{t=0}^{T}$, it follows from (3.4) that $\lim _{n \rightarrow \infty} \mathrm{P}\left(C_{n}\right)=0$. This together with (3.5) gives, for sufficiently large $n$, $\sup _{\tau \in \mathcal{T}_{[0, T]}^{W}} \mathrm{E}^{W}\left[\left(Y^{W}(\tau)-Q^{(n)}(\tau) / M(\tau)\right)^{+}\right]<R(x)+\varepsilon$, as required.

Given a probability space $(\Omega, \mathcal{F}, \mathrm{P})$, consider a càdlàg stochastic process $S=\left\{S_{t}: \Omega \rightarrow\right.$ $\left.\mathbb{R}^{d}\right\}_{t=0}^{\Theta}, \Theta<\infty$. Denote by $\mathcal{F}^{S}=\left\{\mathcal{F}_{t}^{S}\right\}_{t=0}^{\Theta}$ the usual filtration of $S$ i.e. the smallest rightcontinuous filtration with respect to which $S$ is adapted, and such that the $\sigma$-algebras contain the null sets. Let $\mathcal{T}_{[0, \Theta]}^{S}$ be the set of all stopping times with respect to $\mathcal{F}^{S}$ which take values in $[0, \Theta]$. 
In [12] the authors introduced the Meyer-Zheng (MZ) topology on the space $\mathbb{D}([0, \Theta] ; \mathbb{R})$. This topology will be denoted by $(\mathbb{D}([0, \Theta] ; \mathbb{R}), \mathrm{MZ})$. The MZ topology is in fact the topology of convergence in measure, it is weaker than the Skorokhod topology, but, for the MZ topology, any sequence of positive uniformly $L^{1}$-bounded supermartingales is relatively compact (see [12]). This fact together with the following lemma will be essential in the proof of Theorem 2.1.

Lemma 3.3. Let $(\Omega, \mathcal{F}, \mathrm{P})$ be a probability space, and let $S^{(n)}: \Omega \rightarrow\left(D[0, \Theta] ; \mathbb{R}^{d}\right)$ be a sequence of stochastic processes such that $S^{(n)} \rightarrow S$ a.s. on the space $\left(\mathbb{D}\left([0, \Theta] ; \mathbb{R}^{d}\right), \diamond\right)$. Assume that, for any $n,\left\{V^{(n)}(t)\right\}_{t=0}^{\Theta}$ is a (one-dimensional) càdlàg, positive supermartingale with respect to the filtration $\mathcal{F}_{[0, \Theta]}^{S_{n}}$, and that $V^{(n)} \rightarrow V$ a.s. on the space $(\mathbb{D}([0, \Theta] ; \mathbb{R}), \mathrm{MZ})$ with respect to the $M Z$ topology. Set

$$
Q(t)=\mathrm{E}\left[V(t) \mid \mathcal{F}_{t}^{S}\right], \quad t \leq \Theta .
$$

Then the process $\{Q(t)\}_{0 \leq t<\Theta}$ is a càdlàg, positive supermartingale with respect to the filtration $\mathcal{F}^{S}$.

Proof. First, let us show that $\{Q(t)\}_{0 \leq t<\Theta}$ is a supermartingale, i.e. for any $s<t<\Theta$ and $D \in \mathcal{F}_{S}^{S}$,

$$
\mathrm{E} \mathbf{1}_{D} V(s) \geq \mathrm{E} \mathbf{1}_{D} V(t) .
$$

Choose $s<s^{\prime}<t, c>0$, and $0<\varepsilon<\min \left(s^{\prime}-s, \Theta-t\right)$. Let $0 \leq \phi \in C\left(\left(\mathbb{D}\left([0, \Theta] ; \mathbb{R}^{d}\right), \S\right)\right)$ be a continuous bounded function such that $\phi(x)$ depends only on the restriction of $x$ to the interval $\left[0, s^{\prime}\right]$. From the definition of the MZ topology we obtain

$$
\begin{aligned}
& \limsup _{n \rightarrow \infty} \mathrm{E} \int_{u=0}^{\varepsilon}\left|\phi\left(S^{(n)}\right)\left(V^{(n)}\left(s^{\prime}+u\right) \wedge c\right)-\phi(S)\left(V\left(s^{\prime}+u\right) \wedge c\right)\right| \mathrm{d} u \\
& \leq|| \phi \|_{\infty} \limsup _{n \rightarrow \infty} \mathrm{E} \int_{u=0}^{\varepsilon}\left(\left|V^{(n)}\left(s^{\prime}+u\right)-V\left(s^{\prime}+u\right)\right| \wedge c\right) \mathrm{d} u \\
& \quad+c \limsup _{n \rightarrow \infty} \mathrm{E} \int_{u=0}^{\varepsilon}\left|\phi(S)-\phi\left(S^{(n)}\right)\right| \mathrm{d} u \\
& =0 .
\end{aligned}
$$

Thus,

$$
\lim _{n \rightarrow \infty} \frac{1}{\varepsilon} \mathrm{E} \int_{u=0}^{\varepsilon} \phi\left(S^{(n)}\right)\left(V^{(n)}\left(s^{\prime}+u\right) \wedge c\right) \mathrm{d} u=\frac{1}{\varepsilon} \mathrm{E} \int_{u=0}^{\varepsilon} \phi(S)\left(V\left(s^{\prime}+u\right) \wedge c\right) \mathrm{d} u .
$$

Similarly,

$$
\lim _{n \rightarrow \infty} \frac{1}{\varepsilon} \mathrm{E} \int_{u=0}^{\varepsilon} \phi\left(S^{(n)}\right)\left(V^{(n)}(t+u) \wedge c\right) \mathrm{d} u=\frac{1}{\varepsilon} \mathrm{E} \int_{u=0}^{\varepsilon} \phi(S)(V(t+u) \wedge c) \mathrm{d} u .
$$

For any $n,\left\{V^{(n)}(\alpha) \wedge c\right\}_{\alpha=0}^{U}$ is a supermartingale with respect to $\mathcal{F}_{[0, \Theta]}^{S^{(n)}}$; this together with (3.7) and (3.8) gives

$$
\frac{1}{\varepsilon} \mathrm{E} \int_{u=0}^{\varepsilon} \phi(S)(V(t+u) \wedge c) \mathrm{d} u \leq \frac{1}{\varepsilon} \mathrm{E} \int_{u=0}^{\varepsilon} \phi(S)\left(V\left(s^{\prime}+u\right) \wedge c\right) \mathrm{d} u .
$$

By taking $\varepsilon \downarrow 0$ we obtain $\mathrm{E} \phi(S)(V(t) \wedge c) \leq \mathrm{E} \phi(S)\left(V\left(s^{\prime}\right) \wedge c\right)$. From density arguments and the fact that $D \in \sigma\left\{S_{u} \mid u<s^{\prime}\right\}$, it follows that $\mathrm{E} \mathbf{1}_{D}\left(V\left(s^{\prime}\right) \wedge c\right) \geq \mathrm{E} \mathbf{1}_{D}(V(t) \wedge c)$, and, 
by letting $s^{\prime} \downarrow s$ and $c \uparrow \infty$, we obtain (3.6). Finally, since the map $t \rightarrow \mathrm{E} Q(t)=\mathrm{E} V(t)$ is right continuous, we find (see [11]) that $Q$ has a càdlàg modification.

In [8] it was proved that

$$
\left(S^{\xi, n}, M^{(n)}\right) \Rightarrow\left(S^{W}, M\right) \quad \text { on the space }\left(\mathbb{D}\left([0, T] ; \mathbb{R}^{d}\right), \S\right) \times(\mathbb{D}([0, T] ; \mathbb{R}), \S) .
$$

We use the notation $S^{(n)} \Rightarrow S$ to indicate that the sequence $S^{(n)}, n \geq 1$, converges weakly to $S$ (see [2, Chapter 1]). We will use the concept of 'extended weak convergence', which was introduced in Aldous [1, Chapter 5]. The original definition was via prediction processes. For the case where the stochastic processes are considered with respect to their usual filtration, Aldous proved that the extended weak convergence is equivalent to a more elementary condition which does not require the use of prediction processes (see [1, Proposition 16.15]). We will use the above condition as the definition of extended weak convergence.

Definition 3.1. A sequence $S^{(n)}: \Omega_{n} \rightarrow \mathbb{D}\left([0, T] ; \mathbb{R}^{d}\right), n \geq 1$, converges in the extended weak sense to a stochastic process $S: \Omega \rightarrow \mathbb{D}\left([0, T] ; \mathbb{R}^{d}\right)$ if, for any $k$ and continuous bounded functions $\psi_{1}, \ldots, \psi_{k} \in C\left(\left(\mathbb{D}\left([0, T] ; \mathbb{R}^{d}\right), \S\right)\right)$,

$$
\left(S^{(n)}, H^{(n, 1)}, \ldots, H^{(n, k)}\right) \Rightarrow\left(S, H^{(1)}, \ldots, H^{(k)}\right) \quad \text { on }\left(\mathbb{D}\left([0, T] ; \mathbb{R}^{d+k}\right), \S\right),
$$

where, for any $t \leq T, 1 \leq i \leq k$, and $n \in \mathbb{N}$,

$$
H_{t}^{(n, i)}=\mathrm{E}_{n}\left[\psi_{i}\left(S^{(n)}\right) \mid \mathcal{F}_{t}^{S^{(n)}}\right], \quad n \in \mathbb{N}, \quad \text { and } \quad H^{(i)}=\mathrm{E}\left[\psi_{i}(S) \mid \mathcal{F}_{t}{ }^{S}\right] .
$$

Here $\mathrm{E}_{n}$ denotes the expectation with respect to the probability measure on $\Omega_{n}$ and $\mathrm{E}$ denotes the expectation with respect to the probability measure on $\Omega$. We will denote extended weak convergence by $S^{(n)} \Rightarrow S$.

Lemma 3.4. We have $S^{\xi, n} \Rightarrow S^{W}$.

Proof. Define the map $G:\left(\mathbb{D}\left([0, T] ; \mathbb{R}^{d}\right), \S\right) \rightarrow\left(\mathbb{D}\left([0, T] ; \mathbb{R}^{d}\right), \S\right)$ by

$$
\left(G\left(x_{1}, \ldots, x_{d}\right)\right)(t)=\left(\operatorname { e x p } \left(x_{1}(t), \ldots, \exp \left(x_{d}(t)\right)\right.\right.
$$

Observe that $G$ is a continuous map with continuous inverse (the inverse is defined only on functions $\left(x_{1}, \ldots, x_{d}\right) \in \mathbb{D}\left([0, T] ; \mathbb{R}^{d}\right)$ which satisfy $\left.\min _{1 \leq i \leq d} \inf _{0 \leq t \leq T} x_{i}(t)>0\right)$. Let

$$
\begin{gathered}
\left\{X(t)=\left(\ln S_{1}^{W}(t), \ldots, \ln S_{d}^{W}(t)\right)\right\}_{t=0}^{T} \\
\text { and } \quad\left\{X^{(n)}(t)=\left(\ln S_{1}^{\xi, n}(t), \ldots, \ln S_{d}^{\xi, n}(t)\right)\right\}_{t=0}^{T}, \quad n \in \mathbb{N} .
\end{gathered}
$$

From (3.9) and the fact that $G$ has a continuous inverse, it follows that $X^{(n)} \Rightarrow X$. For any $n$, the process $X^{(n)}$ has independent increments and the process $X$ is a continuous process with independent increments. From Corollary 2 of [9] we obtain $X^{(n)} \Rightarrow X$, and so (since $G$ is continuous) $S^{\xi, n} \Rightarrow S^{W}$.

Remark 3.1. In [9] the authors dealt with one-dimensional stochastic processes. It can be shown by using the same arguments as in the original proof that the results (Theorem 1 and Corollaries 1-2 of [9]) can be extended to stochastic processes which accept values in $\mathbb{R}^{d}$, $d \in \mathbb{N}$. 


\section{Proof of the main result}

In this section we complete the proof of Theorem 2.1. Fix $x$. We start with the proof of the inequality $R(x) \leq \lim _{n \rightarrow \infty} R_{n}(x)$. Here and in the sequel, for the sake of simplicity, we will assume that indices have been renamed so that the whole sequence converges. Let $\pi_{n} \in \mathcal{A}^{\xi, n}(x), n \in \mathbb{N}$, be a sequence such that

$$
R_{n}\left(\pi_{n}\right)<R_{n}(x)+\frac{1}{n} \text { for all } n \in \mathbb{N} .
$$

For any $n \in \mathbb{N}$, define the stochastic process $\left\{Z^{(n)}(t)\right\}_{t=0}^{2 T}$ by $Z^{(n)}(t)=V^{\pi_{n}}(k) M^{(n)}(t)$ for $k T / n \leq t<(k+1) T / n$ and $k<n$, and by $Z^{(n)}(t)=V^{\pi_{n}}(n) M^{(n)}(T)$ for $t \geq T$. From (2.4), it follows that $\left\{Z^{(n)}(t)\right\}_{t=0}^{2 T}$ is a càdlàg martingale with respect to $\mathrm{P}^{\xi}$ and the filtration $\left\{\mathcal{F}_{t}^{S^{n, \xi}}\right\}_{t=0}^{2 T}$, where we set $\mathcal{F}_{t}^{S^{n, \xi}}=\mathcal{F}_{T}^{S^{n, \xi}}$ for $t \geq T$. From [12], it follows that the sequence $Z^{(n)}, n \in \mathbb{N}$, is tight on the space $(\mathbb{D}([0, T] ; \mathbb{R}), \mathrm{MZ})$. We can extend all the processes in (3.9) to the interval $[0,2 T]$, letting their paths be constants on the interval [T,2T]. From (3.9) we find that the sequence $\left(S^{\xi, n}, M^{(n)}, Z^{(n)}\right), n \in \mathbb{N}$, is tight on the space

$$
\left(\mathbb{D}\left([0,2 T] ; \mathbb{R}^{d}\right), \S\right) \times(\mathbb{D}([0,2 T] ; \mathbb{R}), \S) \times(\mathbb{D}([0,2 T] ; \mathbb{R}), \mathrm{MZ}) .
$$

Thus, there exists a subsequence such that $\left(S^{\xi, n}, M^{(n)}, Z^{(n)}\right) \Rightarrow\left(S^{W}, M, Z\right)$ for some stochastic process $Z$ which satisfies $Z(0) \leq x$. Next, from the Skorokhod representation theorem (see [7]), it follows that, without loss of generality, we can assume that there exists a probability space $(\Omega, \mathcal{F}, \mathrm{P})$ on which

$$
\left(S^{\xi, n}, M^{(n)}, Z^{(n)}\right) \rightarrow\left(S^{W}, M, Z\right) \quad \text { a.s. }
$$

on the space

$$
\left(\mathbb{D}\left([0,2 T] ; \mathbb{R}^{d}\right), \S\right) \times(\mathbb{D}([0,2 T] ; \mathbb{R}), \S) \times(\mathbb{D}([0,2 T] ; \mathbb{R}), \mathrm{MZ}) .
$$

From Lemma 3.3, it follows that the process $Q(t):=\mathrm{E}\left[Z(t) \mid \mathcal{F}_{t}{ }^{W}\right], t \leq T$, is a càdlàg supermartingale. The process $V(t):=(Q(t) \wedge \Gamma(t)) / M(t), t \leq T$, is a càdlàg supermartingale of class $\mathcal{D}$ with respect to the martingale measure $\tilde{\mathrm{P}}^{W}(\Gamma(t)$ was introduced after (3.2)). From Doob's decomposition theorem and the martingale representation theorem, we find that there exists a portfolio $\pi \in \mathcal{A}(x)$ such that

$$
V^{\pi}(0)=V(0) \leq Q(0)=Z(0) \leq x \quad \text { and } \quad V^{\pi}(t) \geq V(t) \quad \text { for all } t \leq T .
$$

From [12], there exists a subsequence $Z^{(n)}$ and a dense set $I \subset[0, T]$ such that, for any $t \in J$,

$$
\lim _{n \rightarrow \infty} Z^{(n)}(t)=Z(t) \quad \text { a.s. }
$$

Choose $\varepsilon>0$. From Lemma 3.1 we find that there exists a stopping time $\tau$ which accepts a finite number of values $\left\{t_{1}<t_{2}<\cdots<t_{m}\right\} \subset I$ such that

$$
R(\pi)<\varepsilon+\mathrm{E}\left[\left(Y^{W}(\tau)-V^{\pi}(\tau)\right)^{+}\right] .
$$

From Lemma 3.2 of [3] and (4.2), it follows that there exists a sequence $\sigma_{n} \in \mathcal{F}_{[0, T]}^{S^{\xi, n}}, n \geq 1$, of stopping times with values in the set $\left\{t_{1}<t_{2}<\cdots<t_{m}\right\}$ which satisfy

$$
\lim _{n \rightarrow \infty} \sigma_{n}=\tau \quad \text { a.s. }
$$


Set $\tau_{n}=\max \left\{k \mid k T / n \leq \sigma_{n}\right\}, n \geq 1$. Observe that, for any $k \leq n,\left\{\tau_{n} \leq k\right\}=\left\{\sigma_{n}<\right.$ $(k+1) T / n\} \in \mathcal{T}_{k}^{\xi}$; thus, for any $n, \tau_{n} \in \mathcal{T}_{n}^{\xi}$. Furthermore,

$$
\left|\frac{\tau_{n} T}{n}-\sigma_{n}\right| \leq \frac{1}{n} \quad \text { and } \quad Z^{(n)}\left(\sigma_{n}\right)=Z^{(n)}\left(\frac{\tau_{n} T}{n}\right) \quad \text { for all } n .
$$

From (2.2), it follows that the random variables $Y^{\xi, n}\left(\tau_{n}\right), n \in \mathbb{N}$, are uniformly integrable. Thus, from Jensen's inequality and (4.1)-(4.7), it follows that

$$
\begin{aligned}
R(x) & \leq R(\pi) \\
& \leq \varepsilon+\mathrm{E}\left[\left(Y^{W}(\tau)-V^{\pi}(\tau)\right)^{+}\right] \\
& \leq \varepsilon+\mathrm{E}\left[\left(Y^{W}(\tau)-\frac{Q(\tau)}{M(\tau)}\right)^{+}\right] \\
& \leq \varepsilon+\mathrm{E}\left[\mathrm{E}\left[Y^{W}(\tau)-\frac{Z(\tau)}{M(\tau)}\right]^{+} \mid \mathcal{F}_{\tau}^{S^{W}}\right] \\
& =\varepsilon+\mathrm{E}\left[\left(Y^{W}(\tau)-\frac{Z(\tau)}{M(\tau)}\right)^{+}\right] \\
& =\varepsilon+\mathrm{E}\left[\lim _{n \rightarrow \infty}\left(Y^{\xi, n}\left(\tau_{n}\right)-\frac{Z^{(n)}\left(\tau_{n} T / n\right)}{M^{(n)}\left(\tau_{n} T / n\right)}\right)^{+}\right] \\
& =\varepsilon+\lim _{n \rightarrow \infty} \mathrm{E}\left[\left(Y^{\xi, n}\left(\tau_{n}\right)-V^{\pi_{n}}\left(\tau_{n}\right)\right)^{+}\right] \\
& \leq \varepsilon+\lim _{n \rightarrow \infty} R_{n}(x) .
\end{aligned}
$$

Since $\varepsilon>0$ was arbitrary, we conclude that $R(x) \leq \lim _{n \rightarrow \infty} R_{n}(x)$.

Next, we show that $R(x) \geq \lim _{n \rightarrow \infty} R_{n}(x)$. Choose $\varepsilon>0$. From Lemma 3.2, it follows that there exists a $0 \leq \psi \in \bar{C}\left(\left(\mathbb{D}\left([0, T] ; \mathbb{R}^{d}\right), 8\right)\right)$ such that the stochastic process $H(t):=$ $\mathrm{E}^{W}\left[\psi\left(S^{W}\right) \mid \mathcal{F}_{t}^{S^{W}}\right], t \leq T$, satisfies $H(0)<x$ and

$$
R(x)>\sup _{\tau \in \mathcal{T}_{[0, T]}^{S W}} \mathrm{E}^{W}\left[\left(Y^{W}(\tau)-\frac{H(\tau)}{M(\tau)}\right)^{+}\right]-\varepsilon .
$$

For any $n$, define the stochastic process $H^{(n)}(t)=\mathrm{E}^{\xi}\left[\psi\left(S^{n, \xi}\right) \mid \mathcal{F}_{t}^{S^{n, \xi}}\right], t \leq T$. From Lemma 3.4 we obtain

$$
\left(S^{\xi, n}, H^{(n)}\right) \Rightarrow\left(S^{W}, H\right) \quad \text { on the space }\left(\mathbb{D}\left([0, T] ; \mathbb{R}^{d}\right), \S\right) \times(\mathbb{D}([0, T] ; \mathbb{R}), \S) .
$$

Since the process $H$ is continuous, $\lim _{n \rightarrow \infty} H^{(n)}(0)=H(0)$. Thus, we will assume that $n$ is sufficiently large such that $H^{(n)}(0) \leq x$. Observe that the process $H^{(n)}(k T / n) / M^{(n)}(k T / n)$, $0 \leq k \leq n$, is a martingale with respect to $\tilde{\mathrm{P}}_{n}^{\xi}$ and the filtration $\left\{\mathcal{F}_{k}^{\xi}\right\}_{k=0}^{n}$; thus (since the multinomial markets are complete), there exists a $\pi_{n}^{\prime} \in \mathcal{A}^{\xi, n}(x)$ such that

$$
V^{\pi_{n}^{\prime}}(k)=\frac{H^{(n)}(k T / n)}{M^{(n)}(k T / n)}, \quad k \leq n .
$$


We find that, for any $n$, there exists a stopping time $\sigma_{n} \in \mathcal{T}_{n}^{\xi}$ which satisfies

$$
\begin{aligned}
\mathrm{E}^{\xi}\left[\left(Y^{\xi, n}\left(\sigma_{n}\right)-\frac{H^{(n)}\left(\sigma_{n} T / n\right)}{M^{(n)}\left(\sigma_{n} T / n\right)}\right)^{+}\right] & >\sup _{\tau \in \mathcal{T}_{n}^{\xi}} \mathrm{E}^{\xi}\left[\left(Y^{\xi, n}(\tau)-\frac{H^{(n)}(\tau T / n)}{M^{(n)}(\tau T / n)}\right)^{+}\right]-\frac{1}{n} \\
& \geq R_{n}\left(\pi_{n}^{\prime}\right)-\frac{1}{n} \\
& \geq R_{n}(x)-\frac{1}{n} .
\end{aligned}
$$

From (3.9) and (4.10), the sequence $\left(S^{\xi, n}, H^{(n)}, M^{(n)}, \sigma_{n} T / n\right)$ is tight on the space

$$
\left(\mathbb{D}\left([0, T] ; \mathbb{R}^{d}\right), \S\right) \times(\mathbb{D}([0, T] ; \mathbb{R}), \S) \times(\mathbb{D}([0, T] ; \mathbb{R}), \S) \times[0, T] .
$$

Thus, there exists a subsequence such that $\left(S^{\xi, n}, H^{(n)}, M^{(n)}, \sigma_{n} T / n\right) \Rightarrow\left(S^{W}, H, M, v\right)$ for some random variable $v \leq T$. From the Skorokhod representation theorem we can assume that there exists a probability space $(\Omega, \mathcal{F}, \mathrm{P})$ on which

$$
\left(S^{\xi, n}, H^{(n)}, M^{(n)}, \frac{\sigma_{n} T}{n}\right) \rightarrow\left(S^{W}, H, M, v\right) \quad \text { a.s. }
$$

on the space $\left(\mathbb{D}\left([0, T] ; \mathbb{R}^{d}\right), \S\right) \times(\mathbb{D}([0, T] ; \mathbb{R}), \S) \times(\mathbb{D}([0, T] ; \mathbb{R}), \S) \times[0, T]$. Observe that the joint distribution of $\left(S^{W}, H, M\right)$ in (4.12) remains as the original one. From Lemma 3.3 of [3], it follows that, for any $t \leq T,\{v \leq t\}$ and $\mathcal{F}_{T}^{S^{W}}$ are conditionally independent given $\mathcal{F}_{t} S^{W}$, and, for any uniformly integrable càdlàg stochastic process $\{\Phi(t)\}_{t=0}^{T}$ adapted to the filtration $\mathcal{F}_{[0, T]}^{S^{W}}$,

$$
\mathrm{E} \Phi(v) \leq \sup _{\tau \in \mathcal{T}_{[0, T]}^{S}} \mathrm{E} \Phi(\tau)
$$

Finally, by using (4.13) for the process $\Phi(t):=\left(Y^{W}(t)-H(t) / M(t)\right)^{+},(4.9)$, and (4.11)(4.12), we obtain

$$
\begin{aligned}
\lim _{n \rightarrow \infty} R_{n}(x) & \leq \lim _{n \rightarrow \infty} \mathrm{E}^{\xi}\left[\left(Y^{\xi, n}\left(\sigma_{n}\right)-\frac{H^{(n)}\left(\sigma_{n} T / n\right)}{M^{(n)}\left(\sigma_{n} T / n\right)}\right)^{+}\right] \\
& =\mathrm{E}\left[\lim _{n \rightarrow \infty}\left(Y^{\xi, n}\left(\sigma_{n}\right)-\frac{H^{(n)}\left(\sigma_{n} T / n\right)}{M^{(n)}\left(\sigma_{n} T / n\right)}\right)^{+}\right] \\
& =\mathrm{E}^{W}\left[\left(Y^{W}(v)-\frac{H(v)}{M(v)}\right)^{+}\right] \\
& <R(x)+\varepsilon
\end{aligned}
$$

which completes the proof.

Remark 4.1. An interesting question is whether Theorem 2.1 is valid for the game options introduced in [10]. Let $F, G:[0, T] \times\left(\mathbb{D}\left([0, T] ; \mathbb{R}^{d}\right), \diamond\right) \rightarrow \mathbb{R}_{+}$such that $F \leq G$ satisfy the assumptions following (2.1). Set

$$
H^{W}(t, s)=G\left(t, S^{W}\right) \mathbf{1}_{\{t<s\}}+F\left(s, S^{W}\right) \mathbf{1}_{\{s \leq t\}}, \quad t, s \in[0, T],
$$

and

$$
H^{\xi, n}(k, l)=G\left(\frac{k T}{n}, S^{\xi, n}\right) \mathbf{1}_{\{k<l\}}+F\left(\frac{l T}{n}, S^{\xi, n}\right) \mathbf{1}_{\{l \leq k\}}, \quad n \in \mathbb{N}, 0 \leq k, l \leq n .
$$


The terms $H^{W}(t, s)$ and $H^{\xi, n}(k, l)$ are the payoff functions for the BS model and the $n$-step multinomial model, respectively. For game options, the shortfall risk is defined by (see [4])

$$
R^{(g)}(x)=\inf _{\pi \in \mathcal{A}^{W}} \inf _{\sigma \in \mathcal{T}_{[0, T]}^{W}} \sup _{\tau \in \mathcal{T}_{[0, T]}^{W}} \mathrm{E}^{W}\left[\left(H^{W}(\sigma, \tau)-V^{\pi}(\sigma \wedge \tau)\right)^{+}\right],
$$

and

$$
R_{n}^{(g)}(x)=\inf _{\pi \in \mathcal{A}^{\xi, n}(x)} \min _{\sigma \in \mathcal{T}_{n}^{\xi}} \max _{\tau \in \mathcal{T}_{n}^{\xi}} \mathrm{E}^{\xi}\left[\left(H^{\xi, n}(\sigma, \tau)-V^{\pi}(\sigma \wedge \tau)\right)^{+}\right]
$$

The question is whether the equality $R^{(g)}(x)=\lim _{n \rightarrow \infty} R_{n}^{(g)}(x)$ holds. Following the proof above, it can be shown that $R^{(g)}(x) \geq \lim \sup _{n \rightarrow \infty} R_{n}^{(g)}(x)$. The inequality $R^{(g)}(x) \leq$ $\liminf _{n \rightarrow \infty} R_{n}^{(g)}(x)$ is more difficult to prove because of the additional inf (in (4.14)) which destroys the convexity that was used in (4.8) (by applying Jensen's inequality). At present, it is not clear whether the weak convergence approach can be applied here.

\section{Analysis of the multinomial models}

In this section we provide a dynamical programming algorithm for the shortfall risks and the corresponding optimal portfolios in the multinomial models. Similar analysis was carried out in [4] for game options in multinomial markets with one risky asset.

Definition 5.1. A function $\psi: \mathbb{R}_{+} \rightarrow \mathbb{R}_{+}$is a piecewise linear function vanishing at $\infty$ if there exists a natural number $n$ such that

$$
\psi(y)=\sum_{i=1}^{n} \mathbf{1}_{\left[a_{i}, a_{i+1}\right)}\left(c_{i} y+d_{i}\right)
$$

where $c_{1}, \ldots, c_{n}, d_{1}, \ldots, d_{n} \in \mathbb{R}$, and $a_{1}<a_{2}<\cdots<a_{n+1}<\infty$.

Let $J=\left\{v^{(1)}, \ldots, v^{(d+1)}\right\} \subset \mathbb{R}^{d}$ such that

$$
\operatorname{span}\left\{v^{(1)}, \ldots, v^{(d+1)}\right\}=\mathbb{R}^{d} \quad \text { and, there exist } p_{1}, \ldots, p_{d+1}>0, \quad \sum_{i=1}^{d+1} p_{i} v^{(i)}=0 .
$$

Define the set $K_{J}=\left\{u \in \mathbb{R}^{d} \mid\left\langle u, v^{(i)}\right\rangle \geq-1, i=1, \ldots, d+1\right\}$. Observe that $K_{J}$ is a compact convex set.

Lemma 5.1. Let $\psi_{1}, \ldots, \psi_{d+1}: \mathbb{R}_{+} \rightarrow \mathbb{R}_{+}$be continuous, nonincreasing, and piecewise linear functions vanishing at $\infty$. Define $\psi: \mathbb{R}_{+} \rightarrow \mathbb{R}_{+}$by

$$
\psi(y)=\min _{u \in K_{J}} \sum_{i=1}^{d+1} \psi_{i}\left(y\left(1+\left\langle u, v^{(i)}\right\rangle\right)\right) .
$$

Then $\psi$ is a continuous, nonincreasing, and piecewise linear function vanishing at $\infty$.

Proof. Clearly, $\psi$ is a nonincreasing function. There exists a natural number $n$ such that

$$
\psi_{i}(y)=\sum_{j=1}^{n} \mathbf{1}_{\left[a_{j}, a_{j+1}\right)}\left(c_{j}^{(i)} y+d_{j}^{(i)}\right), \quad i=1, \ldots, d+1,
$$


where $c_{j}^{(i)}, d_{j}^{(i)} \in \mathbb{R}$ and $0=a_{1}<a_{2}<\cdots<a_{n+1}<\infty$. Define $I_{k}=\left[a_{k}, a_{k+1}\right)$, $k=1, \ldots, n$, and $I_{n+1}=\left[a_{n+1}, \infty\right)$. Set $\lambda_{i}=1+\sup _{u \in K}\left\langle u, v^{(i)}\right\rangle, i \leq d+1$. Note that, for any $y_{1}, y_{2} \in \mathbb{R}_{+}$,

$$
\begin{aligned}
\left|\psi\left(y_{1}\right)-\psi\left(y_{2}\right)\right| & \leq \sum_{i=1}^{d+1} \sup _{u \in K}\left|\psi_{i}\left(y_{1}\left(1+\left\langle u, v^{(i)}\right\rangle\right)\right)-\psi_{i}\left(y_{2}\left(1+\left\langle u, v^{(i)}\right\rangle\right)\right)\right| \\
& \leq\left|y_{1}-y_{2}\right| \sum_{i=1}^{d+1} \lambda_{i} \max _{1 \leq j \leq n}\left|c_{j}^{(i)}\right| .
\end{aligned}
$$

Thus, $\psi$ is a continuous function. Next, we prove that $\psi$ is a piecewise linear function. Fix $y>0$, and introduce the set $L_{y}=\left\{a_{1} / y-1, \ldots, a_{n+1} / y-1\right\}$. For any $1 \leq \alpha \leq d+1$ and $\beta \in\{1, \ldots, n+1\}^{d+1}$, define the sets

$$
L_{\alpha}^{(y)}=\left\{w \in \mathbb{R}^{d} \mid\left\langle v^{(i)}, w\right\rangle \in L_{y}, i \in\{1, \ldots, d+1\} \backslash\{\alpha\}\right\}
$$

and

$$
K_{\beta}^{(y)}=\left\{u \in \mathbb{R}^{d} \mid y\left(1+\left\langle v^{(j)}, u\right\rangle\right) \in I_{\beta_{j}} \text { for all } j \leq d+1\right\} .
$$

Set $L^{(y)}=\bigcup_{\alpha=1}^{d+1} L_{\alpha}^{(y)}$. There exists a finite sequence of real numbers $c_{1}, \ldots, c_{m}, e_{1}, \ldots, e_{m}$ (which does not depend on $y$ ) such that any $v \in L^{(y)}$ is of the form $v=\left(c_{k_{1}}+e_{r_{1}} / y, \ldots, c_{k_{d}}+\right.$ $\left.e_{r_{d}} / y\right), k_{1}, \ldots, k_{d}, r_{1}, \ldots, r_{d} \in\{1, \ldots, m\}$. Note that, for any $\beta, K_{\beta}^{(y)} \subset K_{J}$ is a compact convex set. Furthermore, the extreme points of $K_{\beta}^{(y)}$ are in $L^{(y)}$. For each $\beta \in\{1, \ldots, n+1\}^{d+1}$, the function $\psi^{(y)}: K_{\beta}^{(y)} \rightarrow \mathbb{R}_{+}$, which is given by $\psi^{(y)}(u)=\sum_{i=1}^{d+1} \psi_{i}\left(y\left(1+\left\langle v^{(i)}, u\right\rangle\right)\right)$, is an affine function. Since $\bigcup_{\beta \in\{1, \ldots, n+1\}^{d+1}} K_{\beta}^{(y)}=K_{J}$, we obtain

$$
\begin{aligned}
\psi(y) & =\min _{\beta \in\{1, \ldots, n+1\}^{d+1}} \min _{u \in K_{\beta}^{(y)}} \psi^{(y)}(u) \\
& =\min _{\beta \in\{1, \ldots, n+1\}^{d+1}} \min _{u \in K_{\beta}^{(y)} \cap L^{(y)}} \psi^{(y)}(u) \\
& =\min _{u \in K_{J} \cap L^{(y)}} \psi^{(y)}(u) .
\end{aligned}
$$

Thus, there exists a finite sequence of real numbers $f_{1}, \ldots, f_{\tilde{m}}, g_{1}, \ldots, g_{\tilde{m}}$ such that, for any $y>0$,

$$
\psi(y)=f_{i} y+g_{i}
$$

for some $i$ (which depends on $y$ ). From this together with the inequality $\psi(y) \leq \sum_{i=1}^{d+1} \psi_{i}(y)$ and the fact that $\psi$ is a continuous function, it follows that $\psi$ is a piecewise linear function vanishing at $\infty$.

Next, fix $n$ and consider the $n$-step multinomial model. For any $\pi \in \mathcal{A}^{\xi, n}$, define a sequence of random variables $\left\{U^{\pi}(k)\right\}_{k=0}^{n}$ by

$$
U^{\pi}(n)=\left(Y^{\xi, n}(n)-V^{\pi}(n)\right)^{+},
$$

and, for $k<n$,

$$
U^{\pi}(k)=\max \left(\mathrm{E}^{\xi}\left[U^{\pi}(k+1) \mid \mathcal{F}_{k}^{\xi}\right],\left(Y^{\xi, n}(k)-V^{\pi}(k)\right)^{+}\right) .
$$


Applying standard results for optimal stopping (see [14, Chapter 1]) to the process $\left(Y^{\xi, n}(k)-\right.$ $\left.V^{\pi}(k)\right)^{+}, k=0,1, \ldots, n$, we obtain

$$
U^{\pi}(0)=\max _{\tau \in \mathcal{T}_{n}^{\xi}} \mathrm{E}^{\xi}\left[\left(Y^{\xi, n}(\tau)-V^{\pi}(\tau)\right)^{+}\right]=R_{n}(\pi) .
$$

Set

$$
\begin{array}{cc}
w^{(i)}=\sqrt{d+1}\left(A_{i 1}, \ldots, A_{i d}\right), & w^{n, i}=\frac{T}{n} b+\sqrt{\frac{T}{n}} w^{(i)} \sigma^{\top}, \quad i \leq d+1, \\
J=\left\{w^{(1)}, \ldots, w^{(d+1)}\right\}, & \text { and } \quad J_{n}=\left\{w^{n, 1}, \ldots, w^{n, d+1}\right\},
\end{array}
$$

where the matrix $A$ and the vector $b$ were introduced in Section 2.

Definition 5.2. Let $0 \leq k<n$, and let $X$ be a nonnegative $\mathcal{F}_{k}^{\xi}$-measurable random variable.

Define the set

$$
\begin{aligned}
& \mathcal{A}_{k}^{(n)}(X)=\left\{Y \mid Y=X\left(1+\left\langle\rho, \frac{T}{n} b+\sqrt{\frac{T}{n}} \xi^{(k+1)} \sigma^{\top}\right\rangle\right)\right. \\
&\left.\rho: \Omega_{\xi} \rightarrow K_{J_{n}} \text { is } \mathcal{F}_{k}^{\xi} \text {-measurable }\right\} .
\end{aligned}
$$

Note that if $V^{\pi}(k)=X$ and $V^{\pi}(k+1)=Y$ for some $\pi=(\gamma(1), \ldots, \gamma(n)) \in \mathcal{A}^{\xi, n}$ and $k<n$, then, from (2.3) and (2.5), $Y=X\left(1+\left\langle\rho, T b / n+(\sqrt{T / n}) \xi^{(k+1)} \sigma^{\top}\right\rangle\right)$, where

$$
\rho=\frac{\mathbf{1}_{\{X>0\}}}{X}\left(\gamma_{1}(k+1) S_{1}^{\xi, n}\left(\frac{k T}{n}\right), \ldots, \gamma_{d}(k+1) S_{d}^{\xi, n}\left(\frac{k T}{n}\right)\right) .
$$

Clearly, if $X=0$ then ( $\pi$ is admissible) $Y=0$. Since we require $Y \geq 0$ to be satisfied for all possible values of $\xi^{(k+1)}$, then in view of the independency of $\rho$ and $\xi^{(k+1)}$ we conclude that $\mathcal{A}_{k}^{(n)}(X)$ is the set of all possible portfolio values at time $k+1$ provided that the portfolio value at time $k$ is $X$.

For any $0 \leq k \leq n$, let $\phi_{k}^{(n)}: J^{k} \rightarrow \mathbb{R}_{+}$such that

$$
\phi_{k}^{(n)}\left(\xi^{(1)}, \ldots, \xi^{(k)}\right)=Y^{\xi, n}(k) .
$$

Define a sequence of functions $H_{k}^{(n)}: \mathbb{R}_{+} \times J^{k} \rightarrow \mathbb{R}_{+}, k=0,1, \ldots, n$, by the following backward relations. For any $u^{(1)}, \ldots, u^{(n)} \in J$ and $y \in \mathbb{R}_{+}$,

$$
H_{n}^{(n)}\left(y, u^{(1)}, \ldots, u^{(n)}\right)=\left(\phi_{n}^{(n)}\left(u^{(1)}, \ldots, u^{(n)}\right)-y\right)^{+}
$$

and, for $k<n$,

$$
\begin{aligned}
& H_{k}^{(n)}\left(y, u^{(1)}, \ldots, u^{(k)}\right) \\
& =\max \left(\left(\phi_{k}^{(n)}\left(u^{(1)}, \ldots, u^{(k)}\right)-y\right)^{+}\right. \\
& \left.\left.\quad \frac{1}{d+1} \inf _{u \in K_{J_{n}}} \sum_{i=1}^{d+1} H_{k+1}^{(n)}\left(y\left(1+\left\langle u, \frac{T}{n} b+\sqrt{\frac{T}{n}} w^{(i)} \sigma^{\top}\right\rangle\right), u^{(1)}, \ldots, u^{(k)}, w^{(i)}\right)\right)\right) .
\end{aligned}
$$


Observe that $J_{n}$ (for sufficiently large $n$ ) satisfies (5.1). Thus, from Lemma 5.1, it follows (by backward induction) that, for any $k \leq n$ and $u^{(1)}, \ldots, u^{(k)} \in J, H_{k}^{(n)}\left(\cdot, u^{(1)}, \ldots, u^{(k)}\right)$ is a continuous, nonincreasing, and piecewise linear function vanishing at $\infty$. These facts allow us to define the functions $\left\{h_{k}^{(n)}: \mathbb{R}_{+} \times J^{k} \rightarrow K_{J_{n}}\right\}_{k=0}^{n-1}$ by

$$
\begin{aligned}
& h_{k}^{(n)}\left(y, u^{(1)}, \ldots, u^{(k)}\right) \\
& \quad=\underset{u \in K_{J_{n}}}{\operatorname{argmin}} \sum_{i=1}^{d+1} H_{k+1}^{(n)}\left(y\left(1+\left\langle u, \frac{T}{n} b+\sqrt{\frac{T}{n}} w^{(i)} \sigma^{\top}\right\rangle\right), u^{(1)}, \ldots, u^{(k)}, w^{(i)}\right) .
\end{aligned}
$$

Namely,

$$
\begin{aligned}
& \min _{u \in K_{J_{n}}} \sum_{i=1}^{d+1} H_{k+1}^{(n)}\left(y\left(1+\left\langle u, \frac{T}{n} b+\sqrt{\frac{T}{n}} w^{(i)} \sigma^{\top}\right\rangle\right), u^{(1)}, \ldots, u^{(k)}, w^{(i)}\right) \\
& =\sum_{i=1}^{d+1} H_{k+1}^{(n)}\left(y\left(1+\left\langle h_{k}^{(n)}\left(y, u^{(1)}, \ldots, u^{(k)}\right), \frac{T}{n} b+\sqrt{\frac{T}{n}} w^{(i)} \sigma^{\top}\right\rangle\right), u^{(1)}, \ldots, u^{(k)}, w^{(i)}\right)
\end{aligned}
$$

for any $y \in \mathbb{R}_{+}$and $u^{(1)}, \ldots, u^{(k)} \in J$.

Let $x>0$ be an initial capital. Define $\tilde{\pi}=\tilde{\pi}(n, x) \in \mathcal{A}^{\xi, n}(x)$ by

$$
\begin{gathered}
V^{\tilde{\pi}}(0)=x, \\
V^{\tilde{\pi}}(k+1)=V^{\tilde{\pi}}(k)\left(1+\left\langle h_{k}^{(n)}\left(V^{\tilde{\pi}}(k), \xi^{(1)}, \ldots, \xi^{(k)}\right), \frac{T}{n} b+\sqrt{\frac{T}{n}} \xi^{(k+1)} \sigma^{\top}\right\rangle\right)
\end{gathered}
$$

for $0 \leq k<n$.

Theorem 5.1. For any $n \in \mathbb{N}$ and $x>0$,

$$
R_{n}(x)=R_{n}(\tilde{\pi}(n, x))=H_{0}^{(n)}(x) .
$$

Proof. Fix $n \in \mathbb{N}$ and $x>0$. Let $\pi \in \mathcal{A}^{\xi, n}(x)$ be an arbitrary portfolio. Define $\tilde{\pi}=$ $\tilde{\pi}(n, x)$. First we prove by backward induction that, for any $k \leq n$,

$$
H_{k}^{(n)}\left(V^{\pi}(k), \xi^{(1)}, \ldots, \xi^{(k)}\right) \leq U^{\pi}(k) \quad \text { and } \quad H_{k}^{(n)}\left(V^{\tilde{\pi}}(k), \xi^{(1)}, \ldots, \xi^{(k)}\right)=U^{\tilde{\pi}}(k) .
$$

For $k=n$, we obtain from (5.2) and (5.4)-(5.6) that relations (5.9) hold with equality. Suppose that relations (5.9) hold for $k+1$; we now prove that they hold for $k$. Let $\rho: \Omega_{\xi} \rightarrow K_{J_{n}}$ be an $\mathcal{F}_{k}^{\xi}$ measurable random vector such that $V^{\pi}(k+1)=V^{\pi}(k)\left(1+\left\langle\rho, T b / n+(\sqrt{T / n}) \xi^{(k+1)} \sigma^{\top}\right\rangle\right)$. From the induction assumption we obtain

$$
\begin{aligned}
\mathrm{E}^{\xi}\left[U^{\pi}(k+1) \mid \mathcal{F}_{k}^{\xi}\right] \\
\quad \geq \mathrm{E}^{\xi}\left[H_{k}^{(n)}\left(V^{\pi}(k+1), \xi^{(1)}, \ldots, \xi^{(k)}, \xi^{(k+1)}\right) \mid \mathcal{F}_{k}^{\xi}\right] \\
\quad=\frac{1}{d+1} \sum_{i=1}^{d+1} H_{k+1}^{(n)}\left(V^{\pi}(k)\left(1+\left\langle\rho, \frac{T}{n} b+\sqrt{\frac{T}{n}} w^{(i)} \sigma^{\top}\right\rangle\right), \xi^{(1)}, \ldots, \xi^{(k)}, w^{(i)}\right) \\
\quad \geq \frac{1}{d+1} \inf _{u \in K_{J_{n}}} \sum_{i=1}^{d+1} H_{k+1}^{(n)}\left(V^{\pi}(k)\left(1+\left\langle u, \frac{T}{n} b+\sqrt{\frac{T}{n}} w^{(i)} \sigma^{\top}\right\rangle\right), \xi^{(1)}, \ldots, \xi^{(k)}, w^{(i)}\right) .
\end{aligned}
$$


Define $\tilde{\rho}=h_{k}^{(n)}\left(V^{\tilde{\pi}}(k), \xi^{(1)}, \ldots, \xi^{(k)}\right)$. From (5.7)-(5.8) and the induction assumption, it follows that

$$
\begin{aligned}
\mathrm{E}^{\xi}\left[U^{\tilde{\pi}}\right. & \left.(k+1) \mid \mathcal{F}_{k}^{\xi}\right] \\
& =\mathrm{E}^{\xi}\left[H_{k}^{(n)}\left(V^{\tilde{\pi}}(k+1), \xi^{(1)}, \ldots, \xi^{(k)}, \xi^{(k+1)}\right) \mid \mathcal{F}_{k}^{\xi}\right] \\
& =\frac{1}{d+1} \sum_{i=1}^{d+1} H_{k+1}^{(n)}\left(V^{\tilde{\pi}}(k)\left(1+\left\langle\tilde{\rho}, \frac{T}{n} b+\sqrt{\frac{T}{n}} w^{(i)} \sigma^{\top}\right\rangle\right), \xi^{(1)}, \ldots, \xi^{(k)}, w^{(i)}\right) \\
& =\frac{1}{d+1} \inf _{u \in K_{J_{n}}} \sum_{i=1}^{d+1} H_{k+1}^{(n)}\left(V^{\tilde{\pi}}(k)\left(1+\left\langle u, \frac{T}{n} b+\sqrt{\frac{T}{n}} w^{(i)} \sigma^{\top}\right\rangle\right), \xi^{(1)}, \ldots, \xi^{(k)}, w^{(i)}\right) .
\end{aligned}
$$

Combining (5.2), (5.4)-(5.6), and (5.10)-(5.11), we find that (5.9) holds. Next, by using (5.9) for $k=0$ and (5.3), it follows that, for any $\pi \in \mathcal{A}^{\xi, n}(x)$,

$$
R_{n}(\pi)=U^{\pi}(0) \geq H_{0}^{(n)}\left(V^{\pi}(0)\right) \geq H_{0}^{(n)}(x)=U^{\tilde{\pi}}(0)=R_{n}(\tilde{\pi}) .
$$

Thus, $R_{n}(x)=R_{n}(\tilde{\pi})=H_{0}^{(n)}(x)$, as required.

\section{Acknowledgements}

Part of this work was done during my PhD studies at the Hebrew University. I would like to express my deepest gratitude to my $\mathrm{PhD}$ adviser, Yuri Kifer, for his guidance throughout my graduate studies. I am also very grateful to Martin Schweizer for helping me to present this work. I would like to thank the anonymous referee for carefully reading the manuscript, which helped to improve the paper. During my PhD studies I was partially supported by the ISF under grant no. 130/06.

\section{References}

[1] Aldous, D. (1981). Weak convergence of stochastic processes for processes viewed in the strasbourg manner. Unpublished manuscript, Statistics Laboratory, University of Cambridge.

[2] Billingsley, P. (1968). Convergence of Probability Measures. John Wiley, New York.

[3] Dolinsky, Y. (2010). Applications of weak convergence for hedging of game options. Ann. Appl. Prob. 20, 1891-1906.

[4] Dolinsky, Y. AND Kifer, Y. (2007). Hedging with risk for game options in discrete time. Stochastics 79, 169-195.

[5] Dolinsky, Y. And Kifer, Y. (2008). Binomial approximations of shortfall risk for game options. Ann. Appl. Prob. 18, 1737-1770.

[6] Dolinsky, Y. AND KIfER, Y. (2010). Binomial approximations for barrier options of Israeli style. To appear in Ann. Dynamic Games.

[7] Dudley, R. M. (1968). Distances of probability measures and random variables. Ann. Math. Statist. 39, 15631572.

[8] HE, H. (1990). Convergence from discrete to continuous time contingent claim prices. Rev. Financial Studies $\mathbf{3}$, 523-546.

[9] JaKubowski, A. AND Slominski, L. (1986). Extended convergence to continuous in probability processes with independent increments. Prob. Theory Relat. Fields 72, 55-82.

[10] KifER, Y. (2000). Game options. Finance Stoch. 4, 443-463.

[11] Liptser, R. S. H. AND ShIRyAeV, A. N. (2001). Statistics of Random Processes. I. Springer, Berlin.

[12] Meyer, P.-A. And Zheng, W. A. (1984). Tightness criteria for laws of semimartingales. Ann. Inst. H. Poincaré Prob. Statist. 20, 353-372.

[13] Mulinacci, S. (2010). The efficient hedging problem for American options. To appear in Finance Stoch.

[14] Peskir, G. And Shiryaev, A. (2006). Optimal Stopping and Free-Boundary Problems. Birkhauser, Basel.

[15] Shiryaev, A. N. (1999). Essentials of Stochastic Finance. World Scientific, River Edge, NJ. 